\title{
State Complexity of Catenation Combined with Star and Reversal* $^{*}$
}

\author{
Bo Cui, Yuan Gao, Lila Kari, and Sheng Yu \\ Department of Computer Science, \\ The University of Western Ontario, \\ London, Ontario, Canada N6A 5B7 \\ \{bcui2, ygao72, lila, syu\}@csd. uwo.ca
}

\begin{abstract}
This paper is a continuation of our research work on state complexity of combined operations. Motivated by applications, we study the state complexities of two particular combined operations: catenation combined with star and catenation combined with reversal. We show that the state complexities of both of these combined operations are considerably less than the compositions of the state complexities of their individual participating operations.
\end{abstract}

\section{Introduction}

It is worth mentioning that in the past 15 years, a large number of papers have been published on state complexities of individual operations, for example, the state complexities of basic operations such as union, intersection, catenation, star, etc. [5, 7, 9, 10, 14, 16, 17, 18], and the state complexities of several other operations such as shuffle, orthogonal catenation, proportional removal, and cyclic shift [2, 3, 4, 11]. However, in practice, it is common that several operations, rather than only a single operation, are applied in a certain order on a number of finite automata. The state complexity of combined operations is certainly an important research direction in state complexity research. The state complexities of a number of combined operations have been studied in the past two years. It has been shown that the state complexity of a combination of several operations are usually not equal to the composition of the state complexities of individual participating operations [6, 12, 13, 15].

In this paper, we study the state complexities of catenation combined with star, i.e., $L_{1} L_{2}^{*}$, and reversal, i.e., $L_{1} L_{2}^{R}$, respectively, where $L_{1}$ and $L_{2}$ are regular languages. These two combined operations are useful in practice. For example, the regular expressions that match URLs can be summarized as $L_{1} L_{2}^{*}$. Also, the state complexity of $L_{1} L_{2}^{R}$ is equal to that of catenation combined with antimorphic involution $\left(L_{1} \theta\left(L_{2}\right)\right)$ in biology. An involution function $\theta$ is such that $\theta^{2}$ equals the identity function. An antimorphic involution is the natural formalization of the notion of Watson-Crick complementarity in biology. Moreover, the combination of catenation and antimorphic involution can naturally formalize a basic biological operation, primer extension. Indeed, the process of creating the Watson-Crick complement of a DNA single strand $w_{1} w_{2}$ uses the enzyme DNA polymerase to extend a known short primer $p=\theta\left(w_{2}\right)$ that is partially complementary to it, to obtain $\theta\left(w_{2}\right) \theta\left(w_{1}\right)=\theta\left(w_{1} w_{2}\right)$. This can be viewed as the catenation between the primer $p$ and $\theta\left(w_{1}\right)$. The reader is referred to [1] for more details about biological definitions and operations.

It has been shown in [18] that (1) the state complexity of the catenation of an $m$-state DFA language (a language accepted by an $m$-state minimal complete DFA) and an $n$-state DFA language is $m 2^{n}-2^{n-1}$,

\footnotetext{
${ }^{*}$ This work is supported by Natural Science and Engineering Council of Canada Discovery Grant R2824A01, Canada Research Chair Award, and Natural Science and Engineering Council of Canada Discovery Grant 41630. All correspondence should be directed to Bo Cui at bcui2@csd.uwo.ca.
} 
(2) the state complexity of the star of a $k$-state DFA language, where the DFA contains at least one final state that is not the initial state, is $2^{k-1}+2^{k-2}$, and (3) the state complexity of the reversal of an $l$-state DFA language is $2^{l}$. In this paper, we show that the state complexities of $L_{1} L_{2}^{*}$ and $L_{1} L_{2}^{R}$ are considerably less than the compositions of their individual state complexities. Let $L_{1}$ and $L_{2}$ be two regular languages accepted by two complete DFAs of sizes $p$ and $q$, respectively. We will show that, if the $q$-state DFA has only one final state which is also its initial state, the state complexity of $L_{1} L_{2}^{*}$ is $p 2^{q}-2^{q-1}$; in the other cases, that is when the $q$-state DFA contains some final states that are not the initial state, the state complexity of $L_{1} L_{2}^{*}$ is $(3 p-1) 2^{q-2}$. This is in contrast to the composition of state complexities of catenation and star that equals $(2 p-1) 2^{2^{q-1}+2^{q-2}-1}$. We will also show that the state complexity of $L_{1} L_{2}^{R}$ is $p 2^{q}-2^{q-1}-p+1$ instead of $p 2^{2^{q}}-2^{2^{q}-1}$, the composition of state complexities of catenation and reversal.

The paper is organized as follows. We introduce the basic notations and definitions used in this paper in the following section. Then, we study the state complexities of catenation combined with star and reversal in Sections 3 and 4 respectively. Due to page limitation, we omit the proofs of Lemma 1 Lemma 2, Lemma 3, Lemma4, Theorem 5, and Lemma 5. We also omit the proof of Theorem 2 for the case when $m \geq 2$ and $n \geq 3$. We conclude the paper in Section 5

\section{Preliminaries}

An alphabet $\Sigma$ is a finite set of letters. A word $w \in \Sigma^{*}$ is a sequence of letters in $\Sigma$, and the empty word, denoted by $\lambda$, is the word of 0 length.

An involution $\theta: \Sigma \rightarrow \Sigma$ is a function such that $\theta^{2}=I$ where $I$ is the identity function and can be extended to an antimorphic involution if, for all $u, v \in \Sigma^{*}, \theta(u v)=\theta(v) \theta(u)$. For example, let $\Sigma=$ $\{a, b, c\}$ and define $\theta$ by $\theta(a)=b, \theta(b)=a, \theta(c)=c$, then $\theta(a a b c)=c a b b$. Note that the well-known DNA Watson-Crick complementarity is a particular antimorphic involution defined over the four-letter DNA alphabet, $\Delta=\{A, C, G, T\}$.

A non-deterministic finite automaton (NFA) is a quintuple $A=(Q, \Sigma, \delta, s, F)$, where $Q$ is a finite set of states, $s \in Q$ is the start state, and $F \subseteq Q$ is the set of final states, $\delta: Q \times \Sigma \rightarrow 2^{Q}$ is the transition function. If $|\delta(q, a)| \leq 1$ for any $q \in Q$ and $a \in \Sigma$, then this automaton is called a deterministic finite automaton (DFA). A DFA is said to be complete if $\delta(q, a)$ is defined for all $q \in Q$ and $a \in \Sigma$. All the DFAs we mention in this paper are assumed to be complete. We extend $\delta$ to $Q \times \Sigma^{*} \rightarrow Q$ in the usual way. Then the automaton accepts a word $w \in \Sigma^{*}$ if $\delta(s, w) \cap F \neq \emptyset$. Two states $p, q \in Q$ are equivalent if the following condition holds: $\delta(p, w) \in F$ if and only if $\delta(q, w) \in F$ for all words $w \in \Sigma^{*}$. It is well-known that a language which is accepted by an NFA can be accepted by a DFA, and such a language is said to be regular. The language accepted by a finite automaton $A$ is denoted by $L(A)$. The reader is referred to [8, 19] for more details about regular languages and finite automata.

The state complexity of a regular language $L$, denoted by $s c(L)$, is the number of states of the minimal complete DFA that accepts $L$. The state complexity of a class $S$ of regular languages, denoted by $\operatorname{sc}(S)$, is the supremum among all $s c(L), L \in S$. The state complexity of an operation on regular languages is the state complexity of the resulting language from the operation as a function of the state complexities of the operand languages. For example, we say that the state complexity of the intersection of an $m$-state DFA language and an $n$-state DFA language is exactly $m n$. This implies that the largest number of states of all the minimal complete DFAs that accept the intersection of two languages accepted by two DFAs of sizes $m$ and $n$, respectively, is $m n$, and such languages exist. Thus, in a certain sense, the state complexity of an operation is a worst-case complexity. 


\section{Catenation combined with star}

In this section, we consider the state complexity of catenation combined with star. Let $L_{1}$ and $L_{2}$ be two languages accepted by two DFAs of sizes $m$ and $n$, respectively. We notice that, if the $n$-state DFA has only one final state which is also its initial state, this DFA also accepts $L_{2}^{*}$. Thus, in such a case, an upper bound for the number of states of any DFA that accepts $L_{1} L_{2}^{*}=L_{1} L_{2}$ is given by the state complexity of catenation as $m 2^{n}-2^{n-1}$. We first show that this upper bound is reachable by some DFAs of this form (Lemma 1). Then, we consider the state complexity of $L_{1} L_{2}^{*}$ in the other cases, that is when the $n$-state DFA contains some final states that are not the initial state. We show that, in such cases, the upper bound (Theorem 1) coincides with the lower bound (Theorem 2).

Lemma 1. For any $m \geq 2$ and $n \geq 2$, there exists a DFA $A$ of $m$ states and a DFA $B$ of $n$ states, where $B$ has only one final state that is also the initial state, such that any DFA accepting the language $L(A) L(B)$, which is equal to $L(A) L(B)^{*}$, needs at least $m 2^{n}-2^{n-1}$ states.

Note that, if $n=1$, due to Theorem 3 in [18], for any DFA $A$ of size $m \geq 1$, the state complexity of a DFA accepting $L(A) L(B)\left(L(A) L(B)^{*}\right)$ is $m$.

In the rest of this section, we only consider cases $L_{1} L_{2}^{*}$ where the DFA for $L_{2}$ contains at least one final state that is not the initial state. Thus, the DFA for $L_{2}$ is of size at least 2.

When considering the size of the DFA for $L_{1}$, we notice that, when the size of this DFA is 1 , the state complexity of $L_{1} L_{2}^{*}$ is 1 .

Lemma 2. Let $A$ be a 1 -state DFA and $B$ be a DFA of $n \geq 1$ states. Then, the necessary and sufficient number of states for a DFA to accept $L(A) L(B)^{*}$ is 1 .

Now, we focus on the cases when $m>1$ and $n>1$, and give an upper bound for the state complexity of $L_{1} L_{2}^{*}$.

Theorem 1. Let $A=\left(Q_{1}, \Sigma, \delta_{1}, s_{1}, F_{1}\right)$ be a DFA such that $\left|Q_{1}\right|=m>1$ and $\left|F_{1}\right|=k_{1}$, and $B=$ $\left(Q_{2}, \Sigma, \delta_{2}, s_{2}, F_{2}\right)$ be a DFA such that $\left|Q_{2}\right|=n>1$ and $\left|F_{2}-\left\{s_{2}\right\}\right|=k_{2} \geq 1$. Then, there exists $a$ DFA of at most $m\left(2^{n-1}+2^{n-k_{2}-1}\right)-k_{1} 2^{n-k_{2}-1}$ states that accepts $L(A) L(B)^{*}$.

Proof. We denote $F_{2}-\left\{s_{2}\right\}$ by $F_{0}$. Then, $\left|F_{0}\right|=k_{2} \geq 1$.

We construct a DFA $C=\{Q, \Sigma, \delta, s, F\}$ for the language $L_{1} L_{2}^{*}$, where $L_{1}$ and $L_{2}$ are the languages accepted by DFAs $A$ and $B$, respectively. Intuitively, $C$ is constructed by first constructing a DFA $B^{\prime}$ for accepting $L_{2}^{*}$, then catenating $A$ to this new DFA. Note that, in the construction for $B^{\prime}$, we need to add an additional initial and final state $s_{2}^{\prime}$. By careful examination, we can check that the states of $B^{\prime}$ are state $s_{2}^{\prime}$ and the elements in $P-\{\emptyset\}$, where $P$ is defined in the following. As the state set we choose

$$
\begin{gathered}
Q=\{r \cup p \mid r \in R \text { and } p \in P\}, \text { where } \\
R=\left\{S \mid S=\left\{q_{i}\right\}, \text { if } q_{i} \notin F_{1}, S=\left\{q_{i}, s_{2}^{\prime}\right\}, \text { otherwise, where } q_{i} \in Q_{1}\right\}, \text { and } \\
P=\left\{S \mid S \subseteq\left(Q_{2}-F_{0}\right)\right\} \cup\left\{T \mid T \subseteq Q_{2}, s_{2} \in T, \text { and } T \cap F_{0} \neq \emptyset\right\} .
\end{gathered}
$$

If $s_{1} \notin F_{1}$, the initial state $s$ is $s=\left\{s_{1}\right\} \cup\{\emptyset\}$, otherwise, $s=\left\{s_{1}, s_{2}^{\prime}\right\} \cup\{\emptyset\}$.

The set of final states $F$ is chosen to be $F=\left\{S \in Q \mid S \cap\left(F_{2} \cup\left\{s_{2}^{\prime}\right\}\right) \neq \emptyset\right\}$.

We denote a state in $Q$ as $\left\{q_{i}\right\} \cup G$, where $q_{i} \in Q_{1}$ and $G \subseteq Q_{2} \cup\left\{s_{2}^{\prime}\right\}$. Then, the transition relation $\delta$ is defined as follows:

$$
\delta\left(\left\{q_{i}\right\} \cup G, a\right)=D_{1} \cup D_{2} \cup D_{3} \text {, for any } a \in \Sigma \text {, where }
$$


$D_{1}$ : If $\delta_{1}\left(q_{i}, a\right)=q_{i}^{\prime} \in F_{1}, D_{1}=\left\{q_{i}^{\prime}, s_{2}^{\prime}\right\}$, otherwise, $D_{1}=\left\{q_{i}^{\prime}\right\}$.

$D_{2}$ : If $s_{2}^{\prime} \in G$, then $D_{2}=\left\{\delta_{2}\left(s_{2}, a\right)\right\}$, otherwise, $D_{2}=\emptyset$.

$D_{3}:$ If $G=\emptyset, D_{3}=\emptyset$, otherwise,

$$
D_{3}= \begin{cases}\delta_{2}(G, a) & \text { if } \delta_{2}(G, a) \cap F_{0}=\emptyset \\ \delta_{2}(G, a) \cup\left\{s_{2}\right\} & \text { otherwise. }\end{cases}
$$

We can verify that the DFA $C$ indeed accepts $L_{1} L_{2}^{*}$. It is clear that each state in $Q$ should consist of exactly one state in $Q_{1}$ and the states in one element of $P-\{\emptyset\}$. Moreover, if a state of $Q$ contains a final state of $A$, then this state also contains the state $s_{2}^{\prime}$.

To get an upper bound for the state complexity of catenation combined with star, we should count the number of states of $Q$. However, as we will show in the following, some states in $Q$ are equivalent.

Let us recall the construction for $B^{\prime}$. Note that, in that construction, states $s_{2}^{\prime}$ and $s_{2}$ should reach the same state on any letter in $\Sigma$. Also note that a state of $Q$ contains $s_{2}^{\prime}$ only when it contains a final state of $A$. Moreover, there exist pairs of states, denoted by $\left\{q_{f}, s_{2}^{\prime}, s_{2}\right\} \cup T$ and $\left\{q_{f}, s_{2}^{\prime}\right\} \cup T$, such that $q_{f}$ is a final state of $A$ and $T \subseteq Q_{2} \backslash\left\{s_{2}\right\}$. Then, we show that the two states in each of such pairs are equivalent as follows. For a letter $a \in \Sigma$ and a word $w \in \Sigma^{*}$,

$$
\delta\left(\left\{q_{f}, s_{2}^{\prime}, s_{2}\right\} \cup T, a w\right)=\delta\left(\left\{q_{f}, s_{2}^{\prime}\right\} \cup T, a w\right)=\delta\left(\delta\left(\left\{q_{f}, s_{2}^{\prime}\right\} \cup T, a\right), w\right) .
$$

Note that the equivalent states are only in the set $F_{1} \times\left\{s_{2}^{\prime}\right\} \times\left\{S \mid S \subseteq\left(Q_{2}-F_{0}\right)\right\}$, and we can furthermore partition this set into two sets as

$$
\begin{aligned}
& F_{1} \times\left\{s_{2}^{\prime}\right\} \times\left\{s_{2}\right\} \times\left\{S^{\prime} \mid S^{\prime} \subseteq\left(Q_{2}-F_{0}-\left\{s_{2}\right\}\right)\right\} \cup \\
& F_{1} \times\left\{s_{2}^{\prime}\right\} \times\left\{S^{\prime} \mid S^{\prime} \subseteq\left(Q_{2}-F_{0}-\left\{s_{2}\right\}\right)\right\} .
\end{aligned}
$$

It is easy to see that, for each state in the former set, there exists one and only one equivalent state in the latter set, and vice versa. Thus, the number of equivalent pairs is $k_{1} 2^{n-k_{2}-1}$.

Finally, we calculate the number of inequivalent states of $Q$. Notice that there are $m$ elements in $R$, $2^{n-k_{2}}$ elements in the first term of $P$, and $\left(2^{k_{2}}-1\right) 2^{n-k_{2}-1}$ elements in the second term of $P$. Therefore, the size of $Q$ is $|Q|=m\left(2^{n-1}+2^{n-k_{2}-1}\right)$. Then, after removing one state from each equivalent pair, we obtain the following upper bound

$$
m\left(2^{n-1}+2^{n-k_{2}-1}\right)-k_{1} 2^{n-k_{2}-1} .
$$

Next, we give examples to show that this upper bound can be reached.

Theorem 2. For any integers $m \geq 2$ and $n \geq 2$, there exists a DFA A of $m$ states and a DFA of $n$ states such that any DFA accepting $L(A) L(B)^{*}$ needs at least $m \frac{3}{4} 2^{n}-2^{n-2}$ states.

Proof. We first give an example of two DFAs $A$ and $B$ of sizes $m \geq 2$ and $n=2$, respectively, and we show that the number of states of a DFA accepting $L(A) L(B)^{*}$ reaches the upper bound given in Theorem 1 We use a three-letter alphabet $\Sigma=\{a, b, c\}$. We omit the cases when $n>2$, due to the page limit.

Define $A=\left(Q_{1}, \Sigma, \delta_{1}, q_{0},\left\{q_{m-1}\right\}\right)$, where $Q_{1}=\left\{q_{0}, q_{1}, \ldots, q_{m-1}\right\}$, and the transitions are given as:

- $\delta_{1}\left(q_{i}, a\right)=q_{i+1}, i \in\{0, \ldots, m-2\}, \delta_{1}\left(q_{m-1}, a\right)=q_{0}$, 
- $\delta_{1}\left(q_{i}, b\right)=q_{i+1}, i \in\{0, \ldots, m-3\}, \delta_{1}\left(q_{m-2}, b\right)=q_{0}, \delta_{1}\left(q_{m-1}, b\right)=q_{m-2}$,

- $\delta_{1}\left(q_{i}, c\right)=q_{i+1}, i \in\{0, \ldots, m-3\}, \delta_{1}\left(q_{m-2}, c\right)=q_{0}, \delta_{1}\left(q_{m-1}, c\right)=q_{m-1}$.

Define $B=\left(Q_{2}, \Sigma, \delta_{2}, 0,\{1\}\right)$, where $Q_{2}=\{0,1\}$, and the transitions are given as:

$$
\begin{array}{lll}
\delta_{2}(0, a)=1, & \delta_{2}(0, b)=0, & \delta_{2}(0, c)=0, \\
\delta_{2}(1, a)=0, & \delta_{2}(1, b)=1, & \delta_{2}(1, c)=0 .
\end{array}
$$

Following the construction described in the proof of Theorem 1 we construct a DFA $C=\left(Q_{3}, \Sigma, \delta_{3}, s_{3}, F_{3}\right)$ that accepts $L(A) L(B)^{*}$. Note that set $P$ only contains three elements $P=\{\emptyset,\{0\},\{0,1\}\}$. To prove that $C$ reaches the upper bound, it is sufficient to show that 1) all the states in $Q_{3}$ are reachable from $s_{3}, 2$ ) after merging the equivalent states $\left\{q_{m-1}, 0^{\prime}\right\}$ and $\left\{q_{m-1}, 0^{\prime}, 0\right\}$, the remaining states are pairwise inequivalent.

We first consider the reachability of all the states. It is clear that state $\left\{q_{i}\right\} \cup\{\emptyset\}$, for $i \in\{1, \ldots, m-$ $2\}$, and state $\left\{q_{m-1}, 0^{\prime}\right\} \cup\{\emptyset\}$ are reachable from $s_{3}$ by reading the strings $a^{i}$ and $a^{m-1}$, respectively. Then, on letters $b$ and $c$, we can reach states $\left\{q_{m-2}, 0\right\}$ and $\left\{q_{m-1}, 0^{\prime}, 0\right\}$, respectively, from state $\left\{q_{m-1}, 0^{\prime}\right\}$. Moreover, state $\left\{q_{i}, 0\right\}, i \in\{0, \ldots, m-3\}$, can be reached from state $\left\{q_{m-2}, 0\right\}$ by reading the string $b^{i+1}$. Lastly, state $\left\{q_{i}, 0,1\right\}, i \in\{0, \ldots, m-2\}$, and state $\left\{q_{m-1}, 0^{\prime}, 0,1\right\}$, are reachable from $\left\{q_{m-1}, 0^{\prime}\right\}$ on inputs $a^{i+1}$ and $a^{m}$, respectively.

Since states $\left\{q_{m-1}, 0^{\prime}\right\}$ and $\left\{q_{m-1}, 0^{\prime}, 0\right\}$ are equivalent, we remove state $\left\{q_{m-1}, 0^{\prime}, 0\right\}$ from $Q_{3}$, and show that the rest of the states are pairwise inequivalent. Let $\left\{q_{i}\right\} \cup G$ and $\left\{q_{j}\right\} \cup H$ be two different states in $Q_{3}$ with $0 \leq i \leq j \leq m-1$. There are three cases:

1. $i<j$. Then the string $a^{m-1-i} c$ is accepted by DFA $C$ starting from state $\left\{q_{i}\right\} \cup G$, but it is not accepted starting from state $\left\{q_{j}\right\} \cup H$. Note that, after reading $a^{m-1-i} c$, state $\left\{q_{i}\right\} \cup G$ reaches a state that contains states $q_{m-1}$ and $0^{\prime}$. In contrast, the state reached by $\left\{q_{i}\right\} \cup H$ on the same input does not contain these states. Moreover, the resulting states cannot contain state 1 , since on letter $c, C$ remains in state 0 from state 0 and goes to state 0 from state 1 .

2. $i=j \neq m-1$. Since $P=\{\emptyset,\{0\},\{0,1\}\}$ consists of only three elements, we consider them individually. It is obvious that, state $\left\{q_{i}, 0,1\right\}$ is not equivalent to either $\left\{q_{i}\right\}$ or $\left\{q_{i}, 0\right\}$, since it is a final state but the latter two are not. States $\left\{q_{i}\right\}$ and $\left\{q_{i}, 0\right\}$ are inequivalent, since on the string $a b$ we can reach a final state from state $\left\{q_{i}, 0\right\}$ but not from state $\left\{q_{i}\right\}$.

3. $i=j=m-1$. There are only two states $\left\{q_{m-1}, 0^{\prime}\right\}$ and $\left\{q_{m-1}, 0^{\prime}, 0,1\right\}$. They are inequivalent, because after reading a letter $b$, state $\left\{q_{m-1}, 0^{\prime}, 0,1\right\}$ leads to a final state of $C$ but $\left\{q_{m-1}, 0^{\prime}\right\}$ does not.

Due to 1) and 2), DFA $C$ has at least $3 m+2$ pairwise inequivalent reachable states, which reaches the upper bound in Theorem 1.

\section{Catenation combined with reversal}

In this section, we first show that the state complexity of catenation combined with an antimorphic involution $\theta\left(L_{1} \theta\left(L_{2}\right)\right)$ is equal to that of catenation combined with reversal. That is, we show, for two regular languages $L_{1}$ and $L_{2}$, that $s c\left(L_{1} \theta\left(L_{2}\right)\right)=s c\left(L_{1} L_{2}^{R}\right)$ (Corollary 1 ). Then, we obtain the state complexity of $L_{1} L_{2}^{R}$ by proving that its upper bound (Theorem 3 ) coincides with its lower bound (Theorem 4 , Theorem [5, and Lemma 5].

We note that an antimorphic involution $\theta$ can be simulated by the composition of two simpler operations: reversal and a mapping $\phi$, which is defined as $\phi(a)=\theta(a)$ for any letter $a \in \Sigma$, and $\phi(u v)=\phi(u) \phi(v)$ where $u, v \in \Sigma^{+}$. Thus, for a language $L$, we have $\theta(L)=\phi\left(L^{R}\right)$ and $\theta(L)=(\phi(L))^{R}$. It is clear that $\phi$ is a homomorphism. Thus, the language resulting from applying such a mapping to a 
regular language remains to be regular. Moreover, we can obtain a relationship between the sizes of the two DFAs that accept $L$ and $\phi(L)$, respectively.

Lemma 3. Let $L \subseteq \Sigma^{*}$ be a language that is accepted by a minimal DFA of size $n, n \geq 1$. Then, the necessary and sufficient number of states of a DFA to accept $\phi(L)$ is $n$.

In order to show that the state complexity of $L_{1} \theta\left(L_{2}\right)$ is equal to that of $L_{1} L_{2}^{R}$, we first show that the state complexity of catenation combined with $\phi$ is equal to that of catenation, i.e., for two regular languages $L_{1}$ and $L_{2}, s c\left(L_{1} \phi\left(L_{2}\right)\right)=s c\left(L_{1} L_{2}\right)$. Due to the above lemma, if $L_{2}$ is accepted by a DFA of size $n, \phi\left(L_{2}\right)$ is accepted by another DFA of size $n$ as well. Thus, the upper bound for the number of states of any DFA that accepts $L_{1} \phi\left(L_{2}\right)$ is clearly less than or equal to $m 2^{n}-2^{n-1}$. The next lemma shows that this upper bound can be reached by some languages.

Lemma 4. For integers $m \geq 1$ and $n \geq 2$, there exist languages $L_{1}$ and $L_{2}$ accepted by two DFAs of sizes $m$ and $n$, respectively, such that any DFA accepting $L_{1} \phi\left(L_{2}\right)$ needs at least $m 2^{n}-2^{n-1}$ states.

As a consequence, we obtain that the state complexity of catenation combined with $\phi$ is equal to that of catenation.

Corollary 1. For two regular languages $L_{1}$ and $L_{2}, s c\left(L_{1} \phi\left(L_{2}\right)\right)=s c\left(L_{1} L_{2}\right)$.

Then, we can easily see that the state complexity of catenation combined with $\theta$ is equal to that of catenation combined with reversal as follows.

$$
s c\left(L_{1} \theta\left(L_{2}\right)\right)=s c\left(L_{1} \phi\left(L_{2}^{R}\right)\right)=s c\left(L_{1} L_{2}^{R}\right) .
$$

In the following, we study the state complexity of $L_{1} L_{2}^{R}$ for regular languages $L_{1}$ and $L_{2}$. We will first look into an upper bound of this state complexity.

Theorem 3. For two integers $m, n \geq 1$, let $L_{1}$ and $L_{2}$ be two regular languages accepted by an $m$-state DFA with $k_{1}$ final states and an $n$-state DFA with $k_{2}$ final states, respectively. Then there exists a DFA of at most $m 2^{n}-k_{1} 2^{n-k_{2}}\left(2^{k_{2}}-1\right)-m+1$ states that accepts $L_{1} L_{2}^{R}$.

Proof. Let $M=\left(Q_{M}, \Sigma, \delta_{M}, s_{M}, F_{M}\right)$ be a DFA of $m$ states, $k_{1}$ final states and $L_{1}=L(M)$. Let $N=$ $\left(Q_{N}, \Sigma, \delta_{N}, s_{N}, F_{N}\right)$ be another DFA of $n$ states, $k_{2}$ final states and $L_{2}=L(N)$. Let $N^{\prime}=\left(Q_{N}, \Sigma, \delta_{N^{\prime}}, F_{N},\left\{s_{N}\right\}\right)$ be an NFA with $k_{2}$ initial states. $\delta_{N^{\prime}}(p, a)=q$ if $\delta_{N}(q, a)=p$ where $a \in \Sigma$ and $p, q \in Q_{N}$. Clearly,

$$
L\left(N^{\prime}\right)=L(N)^{R}=L_{2}^{R} .
$$

After performing subset construction on $N^{\prime}$, we can get an equivalent, $2^{n}$-state DFA $A=\left(Q_{A}, \Sigma, \delta_{A}, s_{A}, F_{A}\right)$ such that $L(A)=L_{2}^{R}$. Please note that $A$ may not be minimal and since $A$ has $2^{n}$ states, one of its final state must be $Q_{N}$. Now we construct a DFA $B=\left(Q_{B}, \Sigma, \delta_{B}, s_{B}, F_{B}\right)$ accepting the language $L_{1} L_{2}^{R}$, where

$$
\begin{aligned}
Q_{B} & =\left\{\langle i, j\rangle \mid i \in Q_{M}, j \in Q_{A}\right\}, \\
s_{B} & =\left\langle s_{M}, \emptyset\right\rangle, \text { if } s_{M} \notin F_{M} ; \\
& =\left\langle s_{M}, F_{N}\right\rangle, \text { otherwise, } \\
F_{B} & =\left\{\langle i, j\rangle \in Q_{B} \mid j \in F_{A}\right\}, \\
\delta_{B}(\langle i, j\rangle, a) & =\left\langle i^{\prime}, j^{\prime}\right\rangle, \text { if } \delta_{M}(i, a)=i^{\prime}, \delta_{A}(j, a)=j^{\prime}, a \in \Sigma, i^{\prime} \notin F_{M} ; \\
& =\left\langle i^{\prime}, j^{\prime} \cup F_{N}\right\rangle, \text { if } \delta_{M}(i, a)=i^{\prime}, \delta_{A}(j, a)=j^{\prime}, a \in \Sigma, i^{\prime} \in F_{M} .
\end{aligned}
$$

It is easy to see that $\delta_{B}\left(\left\langle i, Q_{N}\right\rangle, a\right) \in F_{B}$ for any $i \in Q_{M}$ and $a \in \Sigma$. This means all the states (two-tuples) ending with $Q_{N}$ are equivalent. There are $m$ such states in total. 
On the other hand, since NFA $N^{\prime}$ has $k_{2}$ initial states, the states in $B$ starting with $i \in F_{M}$ must end with $j$ such that $F_{N} \subseteq j$. There are in total $k_{1} 2^{n-k_{2}}\left(2^{k_{2}}-1\right)$ states which don't meet this.

Thus, the number of states of the minimal DFA accepting $L_{1} L_{2}^{R}$ is no more than

$$
m 2^{n}-k_{1} 2^{n-k_{2}}\left(2^{k_{2}}-1\right)-m+1 .
$$

This result gives an upper bound for the state complexity of $L_{1} L_{2}^{R}$. Next we show that this bound is reachable.

Theorem 4. Given two integers $m \geq 2, n \geq 2$, there exists a DFA $M$ of $m$ states and a DFA $N$ of $n$ states such that any DFA accepting $L(M) L(N)^{R}$ needs at least $m 2^{n}-2^{n-1}-m+1$ states.

Proof. Let $M=\left(Q_{M}, \Sigma, \delta_{M}, 0,\{m-1\}\right)$ be a DFA, where $Q_{M}=\{0,1, \ldots, m-1\}, \Sigma=\{a, b, c\}$, and the transitions are given as:

- $\delta_{M}(i, x)=i, i=0, \ldots, m-1, x \in\{a, b\}$,

- $\delta_{M}(i, c)=i+1 \bmod m, i=0, \ldots, m-1$.

Let $N=\left(Q_{N}, \Sigma, \delta_{N}, 0,\{0\}\right)$ be a DFA, where $Q_{N}=\{0,1, \ldots, n-1\}, \Sigma=\{a, b, c\}$, and the transitions are given as:

- $\delta_{N}(0, a)=n-1, \delta_{N}(i, a)=i-1, i=1, \ldots, n-1$,

- $\delta_{N}(0, b)=1, \delta_{N}(i, b)=i, i=1, \ldots, n-1$,

- $\delta_{N}(0, c)=1, \delta_{N}(1, c)=0, \delta_{N}(j, c)=j, j=2, \ldots, n-1$, if $n \geq 3$.

Now we design a DFA $A=\left(Q_{A}, \Sigma, \delta_{A},\{0\}, F_{A}\right)$, where $Q_{A}=\left\{q \mid q \subseteq Q_{N}\right\}, \Sigma=\{a, b, c\}, F_{A}=\{q \mid$ $\left.0 \in q, q \in Q_{A}\right\}$, and the transitions are defined as:

$$
\delta_{A}(p, e)=\left\{j \mid \delta_{N}(j, e)=i, i \in p\right\}, p \in Q_{A}, e \in \Sigma .
$$

It has been shown in [18] that $A$ is a minimal DFA that accepts $L(N)^{R}$. Let $B=\left(Q_{B}, \Sigma=\{a, b, c\}, \delta_{B}, s_{B}=\right.$ $\left.\langle 0, \emptyset\rangle, F_{A}\right)$ be another DFA, where

$$
\begin{aligned}
& Q_{B}=\left\{\langle p, q\rangle \mid p \in Q_{M}-\{m-1\}, q \in Q_{A}-\left\{Q_{N}\right\}\right\} \cup\left\{\left\langle 0, Q_{N}\right\rangle\right\} \\
& \cup\left\{\langle m-1, q\rangle \mid q \in Q_{A}-\left\{Q_{N}\right\},\{0\} \in q\right\}, \\
& F_{B}=\left\{\langle p, q\rangle \mid q \in F_{A},\langle p, q\rangle \in Q_{B}\right\},
\end{aligned}
$$

and for each state $\langle p, q\rangle \in Q_{B}$ and each letter $e \in \Sigma$,

$$
\delta_{B}(\langle p, q\rangle, e)=\left\{\begin{array}{cl}
\left\langle p^{\prime}, q^{\prime}\right\rangle & \text { if } \delta_{M}(p, e)=p^{\prime} \neq m-1, \delta_{A}(q, e)=q^{\prime} \neq Q_{N}, \\
\left\langle p^{\prime}, q^{\prime}\right\rangle & \text { if } \delta_{M}(p, e)=p^{\prime}=m-1, \\
& \delta_{A}(q, e)=r^{\prime}, q^{\prime}=r^{\prime} \cup\{0\}, q^{\prime} \neq Q_{N}, \\
\left\langle 0, Q_{N}\right\rangle & \text { if } \delta_{M}(p, e)=m-1, \delta_{A}(q, e)=r^{\prime}, r^{\prime} \cup\{0\}=Q_{N} \\
\left\langle 0, Q_{N}\right\rangle & \text { if } \delta_{M}(p, e) \neq m-1, \delta_{A}(q, e)=Q_{N}
\end{array}\right.
$$

As we mentioned in last proof, all the states (two-tuples) ending with $Q_{N}$ are equivalent. So here, we replace them with one state: $\left\langle 0, Q_{N}\right\rangle$. And all the states starting with $m-1$ must end with $j \in Q_{A}$ such 
that $0 \in j$. It is easy to see that $B$ accepts the language $L(M) L(N)^{R}$. It has $m 2^{n}-2^{n-1}-m+1$ states. Now we show that $B$ is a minimal DFA.

(I) We first show that every state $\langle i, j\rangle \in Q_{B}$ is reachable by induction on the size of $j$. Let $k=|j|$ and $k \leq n-1$. Note that state $\left\langle 0, Q_{N}\right\rangle$ is reachable from state $\langle 0, \emptyset\rangle$ over string $c^{m} b(a b)^{n-2}$.

When $k=0, i$ should be less than $m-1$ according to the definition of $B$. Then, there always exists a string $w=c^{i}$ such that $\delta_{B}(\langle 0, \emptyset\rangle, w)=\langle i, \emptyset\rangle$.

Basis $(k=1)$ : State $\langle m-1,\{0\}\rangle$ can be reached from state $\langle m-2, \emptyset\rangle$ on a letter $c$. State $\langle 0,\{0\}\rangle$ can be reached from state $\langle m-1,\{0\}\rangle$ on string $c a^{n-1}$. Then, for $i \in\{1, \ldots, m-2\}$, state $\langle i,\{0\}\rangle$ is reachable from state $\langle i-1,\{0\}\rangle$ on string $c a^{n-1}$. Moreover, for $i \in\{0, \ldots, m-2\}$, state $\langle i, j\rangle$ is reachable from state $\langle i,\{0\}\rangle$ on string $a^{j}$.

Induction steps: Assume that all states $\langle i, j\rangle$ such that $|j|<k$ are reachable. Then, we consider the states $\langle i, j\rangle$ where $|j|=k$. Let $j=\left\{j_{1}, j_{2}, \ldots, j_{k}\right\}$ such that $0 \leq j_{1}<j_{2}<\ldots<j_{k} \leq n-1$. We consider the following four cases:

1. $j_{1}=0$ and $j_{2}=1$. State $\left\langle m-1,\left\{0,1, j_{3}, \ldots, j_{k}\right\}\right\rangle$ is reachable from state $\left\langle m-2,\left\{0, j_{3}, \ldots, j_{k}\right\}\right\rangle$ on a letter $c$. Then, for $i \in\{0, \ldots, m-2\}$, state $\langle i, j\rangle$ can be reached from state $\left\langle m-1,\left\{0,1, j_{3}, \ldots, j_{k}\right\}\right\rangle$ on string $c^{i+1}$.

2. $i=0, j_{1}=0$, and $j_{2}>1$. State $\langle 0, j\rangle$ can be reached as follows:

$$
\left\langle 0,\left\{j_{1}, j_{2}, \ldots, j_{k}\right\}\right\rangle=\delta_{B}\left(\left\langle m-2,\left\{j_{3}-j_{2}+1, \ldots, j_{k}-j_{2}+1, n-j_{2}+1\right\}\right\rangle, c^{2} a^{j_{2}-1}\right) .
$$

3. $i=0$ and $j_{1}>0$. State $\langle 0, j\rangle$ is reachable from state $\left\langle 0,\left\{0, j_{2}-j_{1}, \ldots, j_{k}-j_{1}\right\}\right\rangle$ over string $a^{j_{1}}$.

4. We consider the remaining states. For $i \in\{1, \ldots, m-1\}$, state $\langle i, j\rangle$ such that $j_{1}=0$ and $j_{2}>1$ can be reached from state $\left\langle i-1,\left\{1, j_{2}, \ldots, j_{k}\right\}\right\rangle$ on a letter $c$, and, for $i \in\{1, \ldots, m-2\}$, state $\langle i, j\rangle$ such that $j_{1}>0$ is reachable from state $\left\langle i,\left\{0, j_{2}-j_{1}, \ldots, j_{k}-j_{1}\right\}\right\rangle$ over string $a^{j_{1}}$. Recall that we do not have states $\langle i, j\rangle$ such that $i=m-1$ and $j_{1}>0$.

(II) We then show that any two different states $\left\langle i_{1}, j_{1}\right\rangle$ and $\left\langle i_{2}, j_{2}\right\rangle$ in $Q_{B}$ are distinguishable. Let us consider the following three cases:

1. $j_{1} \neq j_{2}$. Without loss of generality, we may assume that $\left|j_{1}\right| \geq\left|j_{2}\right|$. Let $x \in j_{1}-j_{2}$. We don't need to consider the case when $x=0$, because, if $0 \in j_{1}-j_{2}$, then the two states are clearly in different equivalent classes. For $0<x \leq n-1$, there always exists a string $t$ such that $\delta_{B}\left(\left\langle i_{1}, j_{1}\right\rangle, t\right) \in F_{B}$ and $\delta_{B}\left(\left\langle i_{2}, j_{2}\right\rangle, t\right) \notin F_{B}$, where

$$
t= \begin{cases}a^{n-x} & \text { if } i_{2} \neq m-1, j_{1} \neq j_{2} \\ a^{n-x-1} c a & \text { if } i_{2}=m-1, j_{1} \neq j_{2}, n>2 \\ c & \text { if } i_{2}=m-1, j_{1} \neq j_{2}, n=2\end{cases}
$$

Note that, under the second condition, after reading the prefix $a^{n-x-1}$ of $t$, state $n-1$ cannot be in the second component of the resulting state. This is because $x \notin j_{2}$.

Also note that when $n=2, j_{1}, j_{2} \in\left\{Q_{N},\{0\},\{1\}\right\}$, where $Q_{N}=\{0,1\}$. Moreover, when $i_{2}=m-1$, $\left\langle i_{2}, j_{2}\right\rangle$ can only be $\langle m-1,\{0\}\rangle$. Due to the definition of $B$, we have that, for $s \geq 1,\left\langle s, Q_{N}\right\rangle \notin Q_{B}$. Thus, it is easy to see that $\left\langle i_{1}, j_{1}\right\rangle$ is either $\left\langle i_{1},\{1\}\right\rangle$ or $\langle 0,\{0,1\}\rangle$. When $\left\langle i_{1}, j_{1}\right\rangle=\left\langle i_{1},\{1\}\right\rangle, 0 \in j_{1}-j_{2}$, so the two states are distinguishable. When $\left\langle i_{1}, j_{1}\right\rangle=\langle 0,\{0,1\}\rangle$, a string $c$ can distinguish them because $\delta_{B}(\langle 0,\{0,1\}\rangle, c) \in F_{B}$ and $\delta_{B}(\langle m-1,\{0\}\rangle, c) \notin F_{B}$.

2. $j_{1}=j_{2} \neq Q_{N}, i_{1} \neq i_{2}$. Without loss of generality, we may assume that $i_{1}>i_{2}$. In this case, $i_{2} \neq m-1$. Let $x \in Q_{N}-j_{1}$. There always exists a string $u=a^{n-x+1} b c^{m-1-i_{1}}$ such that $\delta_{B}\left(\left\langle i_{1}, j_{1}\right\rangle, u\right) \in F_{B}$ and $\delta_{B}\left(\left\langle i_{2}, j_{2}\right\rangle, u\right) \notin F_{B}$. 
Let $\left\langle i_{1}, j_{1}^{\prime}\right\rangle$ and $\left\langle i_{2}, j_{1}^{\prime}\right\rangle$ be two states reached from states $\left\langle i_{1}, j_{1}\right\rangle$ and $\left\langle i_{2}, j_{2}\right\rangle$ on the prefix $a^{n-x+1}$ of $w$, respectively. We notice that state 1 of $N$ cannot be in $j_{1}^{\prime}$. Then, after reading another letter $b$, we reach states $\left\langle i_{1}, j_{1}^{\prime \prime}\right\rangle$ and $\left\langle i_{2}, j_{1}^{\prime \prime}\right\rangle$, respectively. It is easy to see that states 0 and 1 of $N$ are not in $j_{1}^{\prime \prime}$. Lastly, after reading the remaining string $c^{m-1-i_{1}}$ from state $\left\langle i_{1}, j_{1}^{\prime \prime}\right\rangle$, the first component of the resulting state is the final state of DFA $M$ and therefore its second component contains state 0 of DFA $N$. In contrast, the second component of the resulting state reached from state $\left\langle i_{2}, j_{1}^{\prime \prime}\right\rangle$ on the same string cannot contain state 0 , and hence it is not a final state of $B$. Note that this includes the case that $j_{1}=j_{2}=\emptyset, i_{1} \neq i_{2}$.

3. We don't need to consider the case $j_{1}=j_{2}=Q_{N}$, because there is only one state in $Q_{B}$ which ends with $Q_{N}$. It is $\left\langle 0, Q_{N}\right\rangle$.

Since all the states in $B$ are reachable and pairwise distinguishable, DFA $B$ is minimal. Thus, any DFA accepting $L(M) L(N)^{R}$ needs at least $m 2^{n}-2^{n-1}-m+1$ states.

This result gives a lower bound for the state complexity of $L(M) L(N)^{R}$ when $m, n \geq 2$. It coincides with the upper bound when $k_{1}=1$ and $k_{2}=1$. In the rest of this section, we consider the remaining cases when either $m=1$ or $n=1$. We first consider the case when $m=1$ and $n \geq 3$. We have $L_{1}=\emptyset$ or $L_{1}=\Sigma^{*}$. When $L_{1}=\emptyset$, for any $L_{2}$, a 1 -state DFA always accepts $L_{1} L_{2}^{R}$, since $L_{1} L_{2}^{R}=\emptyset$. The following theorem provides a lower bound for the latter case.

Theorem 5. Given an integer $n \geq 3$, there exists a DFA $M$ of 1 state and a DFA $N$ of $n$ states such that any DFA accepting $L(M) L(N)^{R}$ needs at least $2^{n-1}$ states.

Now, we consider the case when $m=1$ and $n=2$.

Lemma 5. There exists a 1-state DFA $M$ and a 2-state DFA $N$ such that any DFA accepting $L(M) L(N)^{R}$ needs at least 2 states.

Lastly, we consider the case when $m \geq 1$ and $n=1$. When $L_{2}=\emptyset$, for any $L_{1}$, a 1-state DFA always accepts $L_{1} L_{2}^{R}=\emptyset$. When $L_{2}=\Sigma^{*}, L_{1} L_{2}^{R}=L_{1} \Sigma^{*}$, since $\left(\Sigma^{*}\right)^{R}=\Sigma^{*}$. Due to Theorem 3 in [18], which states that, for any DFA $A$ of size $m \geq 1$, the state complexity of $L(A) \Sigma^{*}$ is $m$, the following is immediate.

Corollary 2. Given an integer $m \geq 1$, there exists an $m$-state DFA $M$ and a 1-state DFA $N$ such that any DFA accepting $L(M) L(N)^{R}$ needs at least $m$ states.

After summarizing Theorems 3, 4, and 5, Lemma 5] and Corollary 2, we obtain the state complexity of the combined operation $L_{1} L_{2}^{R}$.

Theorem 6. For any integer $m \geq 1, n \geq 1, m 2^{n}-2^{n-1}-m+1$ states are both necessary and sufficient in the worst case for a DFA to accept $L(M) L(N)^{R}$, where $M$ is an m-state DFA and $N$ is an n-state DFA.

\section{Conclusion}

Motivated by their applications, we have studied the state complexities of two particular combinations of operations: catenation combined with star and catenation combined with reversal. We proved that they are significantly lower than the compositions of the state complexities of their individual participating operations. Thus, this paper shows further that the state complexity of a combination of operations has to be studied individually.

\section{Acknowledgement}

We would like to thank the anonymous referees of DCFS 2010 for their careful reading and valuable suggestions. 


\section{References}

[1] M. Amos: Theoretical and Experimental DNA Computation (Natural Computing Series), Springer, 2005

[2] C. Campeanu, K. Salomaa, S. Yu: Tight lower bound for the state complexity of shuffle of regular languages, Journal of Automata, Languages and Combinatorics 7 (3) (2002) 303-310

[3] M. Daley, M. Domaratzki, K. Salomaa: State complexity of orthogonal catenation, in: Proc. of DCFS 2008, Charlottetown, PE, Canada, July 16-18, 2008, 134-144

[4] M. Domaratzki: State complexity and proportional removals, Journal of Automata, Languages and Combinatorics 7 (2002) 455-468

[5] M. Domaratzki, A. Okhotin: State complexity of power, Theoretical Computer Science 410(24-25) (2009) 2377-2392

[6] Y. Gao, K. Salomaa, S. Yu: The state complexity of two combined operations: star of catenation and star of Reversal, Fundam. Inform. 83 (1-2) (2008) 75-89

[7] M. Holzer, M. Kutrib: State complexity of basic operations on nondeterministic finite automata, in: Proc. of CIAA 2002, LNCS 2608, 2002, 148-157

[8] J. E. Hopcroft, R. Motwani, J. D. Ullman: Introduction to Automata Theory, Languages, and Computation (2nd Edition), Addison Wesley, 2001

[9] J. Jirásek, G. Jirásková, A. Szabari: State complexity of concatenation and complementation of regular languages, Int. J. of Foundations of Computer Science 16 (2005) 511-529

[10] G. Jirásková: State complexity of some operations on binary regular languages, Theoretical Computer Science 330 (2005) 287-298

[11] G. Jirásková, A. Okhotin: State complexity of cyclic shift, in: Proc. of DCFS 2005, Como, Italy, June 30-July 2, 2005, 182-193

[12] G. Jirásková, A. Okhotin: On the state complexity of star of union and star of intersection, Turku Center for Computer Science TUCS Tech. Report No. 825, 2007

[13] G. Liu, C. Martin-Vide, A. Salomaa, S. Yu: State complexity of basic language operations combined with reversal, Inform. and Comput. 206 (2008) 1178-1186

[14] G. Pighizzini, J. O. Shallit: Unary language operations, state complexity and Jacobsthal's function, Int. J. of Foundations of Computer Science 13 (2002) 145-159

[15] A. Salomaa, K. Salomaa, S. Yu: State complexity of combined operations, Theoretical Computer Science 383 (2007) 140-152

[16] A. Salomaa, D. Wood, S. Yu: On the state complexity of reversals of regular languages, Theoretical Computer Science 320 (2004) 293-313

[17] S. Yu: State complexity of regular languages, Journal of Automata, Languages and Combinatorics 6 (2) (2001) 221-234

[18] S. Yu, Q. Zhuang, K. Salomaa: The state complexity of some basic operations on regular languages, Theoretical Computer Science 125 (1994) 315-328

[19] S. Yu: Regular languages, in: G. Rozenberg, A. Salomaa (Eds.), Handbook of Formal Languages, Vol. 1, Springer-Verlag, 1997, 41-110 